\title{
Fractional Chern Insulators with Strong Interactions that Far Exceed Band Gaps
}

\author{
Stefanos Kourtis \\ Institute for Theoretical Solid State Physics, IFW Dresden, 01171 Dresden, Germany \\ Titus Neupert \\ Princeton Center for Theoretical Science, Princeton University, Princeton, New Jersey 08544, USA \\ Claudio Chamon \\ Physics Department, Boston University, Boston, Massachusetts 02215, USA \\ Christopher Mudry \\ Condensed Matter Theory Group, Paul Scherrer Institute, CH-5232 Villigen PSI, Switzerland
}

(Received 28 October 2013; published 27 March 2014)

\begin{abstract}
We study two models for spinless fermions featuring topologically nontrivial bands characterized by Chern numbers $C= \pm 1$ at fractional filling. Using exact diagonalization, we show that, even for infinitely strong nearest-neighbor repulsion, the ground states of these models belong to the recently discovered class of quantum liquids called fractional Chern insulators (FCI). Thus, we establish that FCI states can arise even if interaction strengths are arbitrarily larger than the noninteracting band gap, going beyond the limits in which FCI states have been previously studied. The strong-coupling FCI states, therefore, depart from the usual isolated-band picture that parallels the fractional quantum Hall effect in Landau levels and demonstrate how a topologically ordered state can arise in a truly multiband system.
\end{abstract}

DOI: 10.1103/PhysRevLett.112.126806

PACS numbers: 73.21.-b, 03.65.Vf, 71.27.+a, 73.43.-f

The recently discovered prospect [1-3] of realizing fractional quantum-Hall (FQH) states [4] with the inclusion of short-range interactions in lattice models featuring fractionally filled, topologically nontrivial bands has garnered considerable interest. Apart from a paradigmatic extension of the FQH effect to lattices, these states, called fractional Chern insulators (FCI), arise without an externally applied magnetic field. Hence, they are an important conceptual step toward technological applications. There are already several proposals for the realization of FCI states, some of which involve optical lattices [5], while others are based on known material structures, such as strained or irradiated graphene [6,7], oxide heterostructures [8], or layered multiorbital systems $[9,10]$. The latter category is particularly promising, since the energy scales at which the desired physics emerges is of the order of room temperature.

Since their inception, FCI states have been studied extensively using various numerical and analytical methods $[11,12]$. Of particular interest have been works that emphasize the differences between FCI states and traditional FQH physics. Perhaps the most obvious difference, which was noticed early on, is that Chern bands, unlike Landau levels, have a nonvanishing dispersion. It was first proven by example in Ref. [10] and then substantiated more formally in Refs. [13-16] that this dispersion may actually favor FCI states. Unlike FQH systems, FCI models can be naturally extended to include both spin species, and it has been shown that the resulting time reversal-symmetric models can be hosts of fractional topological insulators
[17], a prospect that was envisaged prior to the advent of FCIs $[18,19]$. Since the Chern number of a band can, in contrast to a Landau level, take values larger than one [20-22], FCI states can occur in partially filled bands with higher Chern numbers [13,23-25]. Finally, topologically ordered states that go markedly beyond the Landau-level picture, in which the topological character is combined with Landau-type order, have been found recently [26].

In the literature on FCIs, most works deal with a single isolated band. The presence of more than one band has been taken into account in few examples $[9,10,26]$, but the effect of band mixing has not been systematically studied. If one wishes to search for FCI states in the laboratory, understanding of how these states can arise in realistic multiband systems is crucial. In this Letter, we wish to pose two fundamental questions for the realization of FCI states, namely (i) whether the mixing of bands by interactions leaves space for FCI states to arise, and (ii) whether FCI states can be found far beyond the energy scale of the band gap. We shall answer both questions positively.

We have studied two prototypical two-sublattice FCI models using exact diagonalization, taking both Chern bands into account. We show that FCI states survive band mixing caused by arbitrarily large interactions. To demonstrate this, we introduce the extreme limit of nearestneighbor interaction going to infinity. In this regime, which can be exploited further in the study of higher dimensional and spinful systems, particles dressed by the interaction form extended objects, which can be interpreted as noninteracting 
hard-core particles occupying more than one lattice site. We find that strong interactions of magnitude far larger than the band gap may actually favor FCI states, regardless of whether the bands are mixed. These observations provide fresh insights into topological ordering, and more importantly, they open prospects to realizing experimentally fractional topological states of matter at high temperatures.

Models. - We consider two models for interacting spinless fermions that hop on a lattice with two inequivalent sites per unit cell. We shall endow both models with topologically nontrivial band structures. They have the general form

$$
\hat{H}:=\hat{H}_{\text {kin }}+\hat{H}_{\text {int }} \text {. }
$$

The kinetic energy $\hat{H}_{\text {kin }}$ is

$$
\hat{H}_{\text {kin }}:=\sum_{k \in \mathrm{BZ}} \hat{\psi}_{k}^{\dagger} \mathcal{H}_{k} \hat{\psi}_{k},
$$

where $\hat{\psi}_{k}^{\dagger} \equiv\left(\hat{c}_{k, A}^{\dagger}, \hat{c}_{k, B}^{\dagger}\right)$ denotes an operator-valued spinor whose upper and lower components create spinless fermions with the wave number $\boldsymbol{k}$ from the Brillouin zone (BZ) on the inequivalent sites $A$ and $B$, respectively. The $2 \times 2$ matrix $\mathcal{H}_{\boldsymbol{k}}$ is

$$
\mathcal{H}_{k}:=g_{0, k} \tau_{0}+g_{k} \cdot \boldsymbol{\tau}+\mu_{\mathrm{s}} \tau_{3},
$$

where we have introduced the $2 \times 2$ unit matrix $\tau_{0}$ together with the three Pauli matrices $\boldsymbol{\tau}=\left(\tau_{1}, \tau_{2}, \tau_{3}\right)$ acting on the indices $A$ and $B$. The functions $g_{0, k}, g_{1, k}, g_{2, k}$, and $g_{3, k}$ are smooth real-valued functions of the wave number $\boldsymbol{k}$ in the thermodynamic limit and we have made explicit the dependence on the staggered chemical potential $\mu_{\mathrm{s}} \in \mathbb{R}$. We shall study two specific examples below, by specifying the functions $g_{\mu, k}$ with $\mu=0,1,2,3$. The interaction term $\hat{H}_{\text {int }}$ is the nearest-neighbor repulsion

$$
\hat{H}_{\mathrm{int}}:=V \sum_{\langle i, j\rangle} \hat{n}_{i} \hat{n}_{j},
$$

where $V \geq 0$ is the strength of the nearest-neighbor repulsion, $\langle\boldsymbol{i}, \boldsymbol{j}\rangle$ are directed nearest-neighbor bonds, and $\hat{n}_{i}$ is the number operator that counts how many spinless fermions occupy the lattice site $i \in \Lambda \equiv \Lambda_{A} \cup \Lambda_{B}$.

The checkerboard-lattice model of Refs. [2,3] can be written as

$$
\begin{gathered}
g_{0, k}=4 t_{3} \cos k_{x} \cos k_{y}, \\
g_{1, k}=4 t \cos \varphi \cos \frac{k_{x}}{2} \cos \frac{k_{y}}{2}, \\
g_{2, k}=4 t \sin \varphi \sin \frac{k_{x}}{2} \sin \frac{k_{y}}{2}, \\
g_{3, k}=2 t_{2}\left(\cos k_{x}-\cos k_{y}\right),
\end{gathered}
$$

where $t, t_{2}$, and $t_{3}$ are first nearest-, second nearest-, and third nearest-neighbor hopping amplitudes, respectively. In this definition, the primitive vectors of the checkerboard lattice have been chosen as $\boldsymbol{a}_{1}=(\sqrt{2} / 2,0)^{\top}$ and $\boldsymbol{a}_{2}=(0, \sqrt{2} / 2)^{\top}$, with the unit-cell sites being at points $(0,0)$ and $(1,1)$. In the following, we will fix $t_{2} / t=0.4$ and $\varphi=\pi / 4$. The flatness of the lower Chern band can be tuned by $t_{3}$ and is maximized at $t_{3} / t \approx 0.3$.

The triangular-lattice model of Refs. $[9,10]$ can be written as

$$
\begin{gathered}
g_{0, \boldsymbol{k}}=2 t_{3} \sum_{j=1}^{3} \cos \left(2 \boldsymbol{k} \cdot \boldsymbol{a}_{j}\right), \\
g_{i, \boldsymbol{k}}=2 t \cos \left(\boldsymbol{k} \cdot \boldsymbol{a}_{i}\right), \quad i=1,2,3,
\end{gathered}
$$

where $\boldsymbol{a}_{1}=(1 / 2,-\sqrt{3} / 2)^{\top}, \quad \boldsymbol{a}_{2}=(1 / 2, \sqrt{3} / 2)^{\top}, \quad$ and $\boldsymbol{a}_{3}=-\left(\boldsymbol{a}_{1}+\boldsymbol{a}_{2}\right)$ are the triangular-lattice unit vectors. The first nearest-neighbor and third nearest-neighbor hopping amplitude are $t$ and $t_{3}$, respectively. The third nearestneighbor hopping amplitude $t_{3}$ can be used to tune the dispersion of the lower Chern band, with the flattest bands achieved for $t_{3} / t \approx 0.2$.

Infinite-V limit.-In the following, we will make use of the limit in which the nearest-neighbor repulsive interaction strength $V$ is taken to infinity. In this case, particles cannot occupy nearest-neighbor sites: any many-body state with two spinless fermions sitting on neighboring sites is projected out of the Hilbert space in this limit.

Thus, for any site $\boldsymbol{i} \in \Lambda$ we define the projected operator $\tilde{c}_{i}^{\dagger}$ by demanding that its action on any state in the occupation basis of the projected Hilbert space is to create a spinless fermion on $\boldsymbol{i}$ if and only if this site and all its nearest-neighbor sites are empty. Otherwise, $\tilde{c}_{i}^{\dagger}$ annihilates any state from the projected Hilbert space. Formally,

$$
\tilde{c}_{i}^{\dagger}:=\hat{c}_{i}^{\dagger} \prod_{j \in\langle i j\rangle}\left(1-\hat{n}_{j}\right),
$$

and

$$
\begin{aligned}
\hat{H}= & t \sum_{\langle i, j\rangle}\left(e^{i \phi_{i, j}} \tilde{c}_{i}^{\dagger} \tilde{c}_{j}+\text { H.c. }\right) \\
& +t_{2} \sum_{\langle\langle i, j\rangle\rangle}(-1)^{|i|}\left(\tilde{c}_{i}^{\dagger} \tilde{c}_{j}+\text { H.c. }\right)+t_{3} \sum_{\left.\left\langle\left\langle i i_{j}\right\rangle\right\rangle\right\rangle}\left(\tilde{c}_{i}^{\dagger} \tilde{c}_{j}+\text { H.c. }\right) \\
& +\mu_{\mathrm{s}} \sum_{i}(-1)^{|i|} \tilde{c}_{i}^{\dagger} \tilde{c}_{i} .
\end{aligned}
$$

Here, $\phi_{i, j}$ are the phase factors needed to represent each of the two models of Eqs. (2) and (3) and we have set $|\boldsymbol{i}|$ to be even (odd) on sublattice $A(B)$. For the triangular-lattice model defined in Eq. (3) with $t_{3}=0$, this limit gives rise to the Hamiltonian

$$
\hat{H}_{\triangle}:=\sum_{\langle i, j\rangle}\left(e^{\mathrm{i} \phi_{i, j}} \tilde{c}_{i}^{\dagger} \tilde{c}_{j}+\text { H.c. }\right) .
$$


Hamiltonian $\hat{H}_{\triangle}$ contains no free parameters. $\hat{H}_{\triangle}$ is similar to previously studied supersymmetric models [27], which yield exotic "superfrustrated" states with extensive groundstate degeneracy in many lattices [28]. Below we shall see that $\hat{H}_{\triangle}$ gives rise to FCI states at $\nu=1 / 3$ of the lower band, i.e., 1/6 filling of the full lattice.

The infinite- $V$ limit comes with a considerable reduction of the dimensionality of the Fock space [29,30]. Technically, this may be crucial in the search for new topological states, especially in higher dimensions, where the lattice coordination and, thus, the reduction of the Hilbert space is typically higher. Evidently, taking furtherneighbor repulsive interactions $V_{2}, V_{3}, \ldots$ to the hard-core limit $V_{2} \rightarrow \infty, V_{3} \rightarrow \infty, \ldots$ allows for even more dramatic reductions of the Hilbert space.

Results and discussion.-The general properties of the two models defined in Eqs. (2) and (3) have been presented elsewhere $[3,10]$. Here, we wish to highlight mainly two points, that may prove to be crucial in the search for FCI states: (i) Band mixing does not necessarily reduce the propensity to form FCI states; (ii) the strength of interactions can be much larger than previously thought and can, in fact, be set to be infinite, without driving the system out of the FCI phase. (We find similar results for $\nu=1 / 5$.)

We will now focus on the filling fraction $\nu=1 / 3$ of the lowest Chern band for each of the two models. We wish to know whether or how the inclusion of $\hat{H}_{\text {int }}$ in $\hat{H}$ will mix the bands formed by the eigenvalues of $\hat{H}_{\text {kin }}$. To this end, we have to measure the contribution-if any-of the upper band to the FCI states, that is, the overlap

$$
n_{+}:=\sum_{k}\left\langle E_{0}\left|\hat{n}_{k,+}\right| E_{0}\right\rangle
$$

where $\left|E_{0}\right\rangle$ is any one of the states in the degenerate ground-state manifold and $\hat{n}_{\boldsymbol{k},+}$ is the operator measuring the density of particles with upper-band character at wave number $\boldsymbol{k}$ (see the Supplemental Material [31]). In Fig. 1, we show the expectation value of $n_{+}$for one of the states in the FCI manifold as a function of inverse interaction strength. We notice that, in the weak-coupling limit, band mixing is very limited. However, as the interaction reaches its maximal value, the mixing increases and saturates at appreciable values for both models. The contributions to the occupation $n_{+}$are almost uniformly distributed across the Brillouin zone in the finite clusters. Even though we cannot reach large enough system sizes for a finite-size extrapolation, $n_{+}$shows no tendency of decreasing upon increasing system size, as can be seen in the inset of Fig. 1. The dependence of $n_{+}$on $V$ is almost identical in the 48 -site cluster with eight particles and the 36-site cluster with six particles.

We will now show that, despite the fact that at $V=\infty$ the bands are mixed, the FCI states remain robust for arbitrarily large interactions. To this end, we present the phase

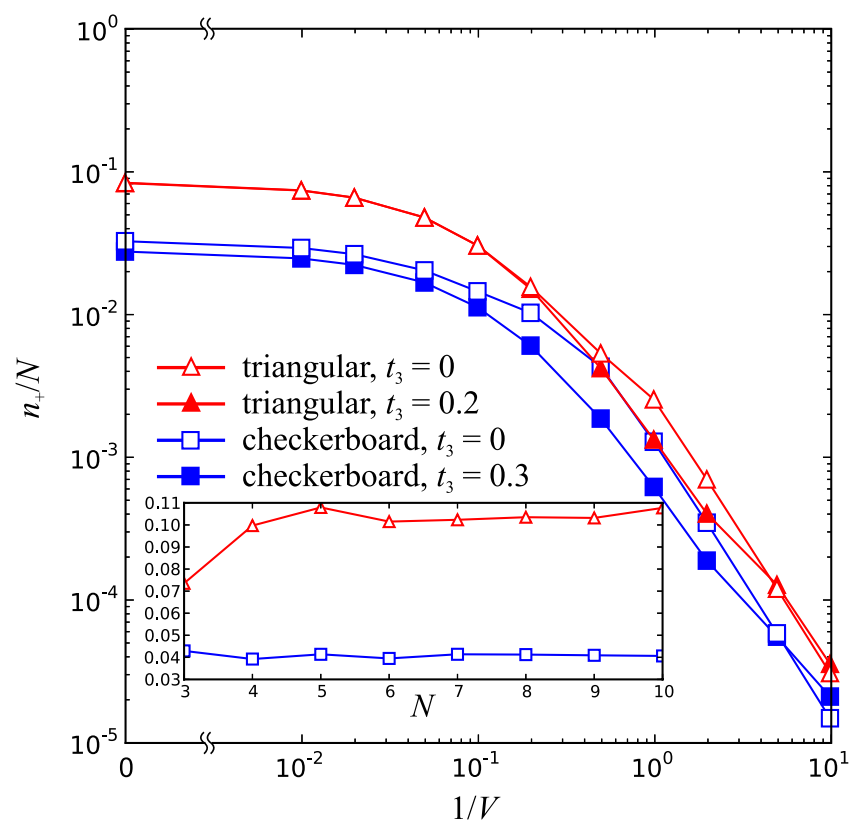

FIG. 1 (color online). The density of upper-band character $n_{+}$ for one of the states in the FCI manifold in the checkerboard (squares) and triangular (triangles) lattice models on a 36-site cluster at $\nu=1 / 3$, with a kinetic term yielding almost flat (full symbols) and nonflat (empty symbols) lower bands, as a function of $1 / V$. Inset: the value of $n_{+}$at $V=\infty$ as a function of system size, parametrized by the number of particles $N$.

diagrams of the two models at $V=\infty$ in the $\mu_{\mathrm{s}}-t_{3}$ plane from Fig. 2. We notice that, in both cases, the FCI phase is quite robust and does not depend crucially on the flatness of the original band. The FCI phase on the triangular lattice is quite sensitive to the introduction of the staggered chemical potential $\mu_{\mathrm{s}}$, presumably because this leads to an effective reduction in dimensionality at low energies. On the contrary, the FCI on the checkerboard lattice seems to be quite robust against $\mu_{\mathrm{s}}$. (This phase seems to survive beyond the
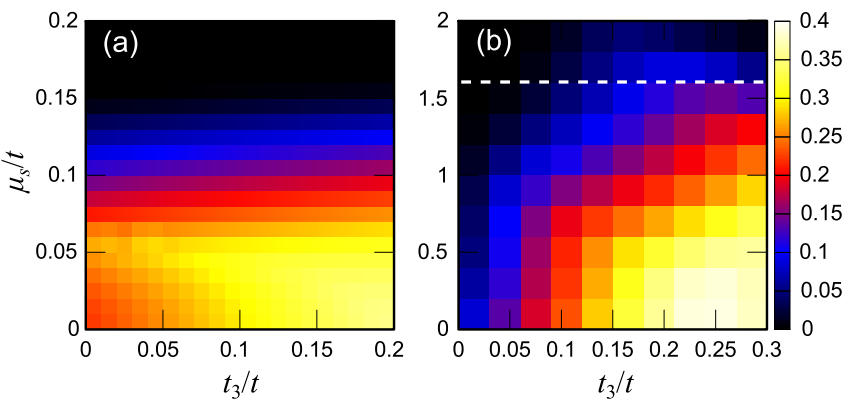

FIG. 2 (color online). Phase diagrams of (a) the triangular and (b) the checkerboard lattice models on a 48-site cluster at $\nu=1 / 3, V=\infty$ in the $\mu_{\mathrm{s}}-t_{3}$ plane. The color coding is the lowest value of the gap between FCI ground states and excited states upon flux insertion (see the Supplemental Material [31]). The dashed white line in (b) denotes the phase boundary for the noninteracting model. 

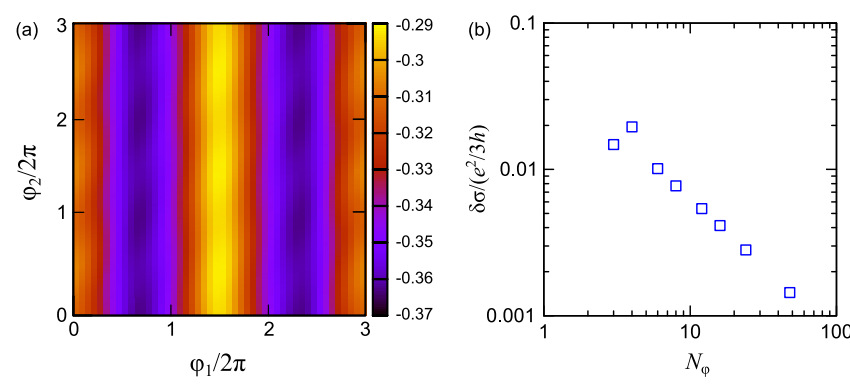

FIG. 3 (color online). (a) Berry curvature and (b) relative error as a function of the square root of the number of points in the flux-Brillouin zone partition for the triangular lattice model on a 48 -site cluster at $\nu=1 / 3, V=\infty, t_{3}=0$.

point where the bands of the noninteracting model would become topologically trivial, but this may be a finite-size artifact.)

It should be mentioned that strong interactions may give rise to competing charge order [10] or more exotic compositely ordered states [26], whenever said competing orders are commensurate with the lattice. The results presented here are, hence, valid for short-range interactions at low enough densities, so that competing strong-coupling instabilities are ruled out.

Within the FCI regime (the colored part of the phase diagrams), the ground-state eigenvalues exhibit the empirical characteristic features of FCI states: threefold degeneracy and spectral flow. In order to establish beyond doubt that the phase is, indeed, an FCI, however, we calculate the Hall conductivity in this regime (see the Supplemental Material [31]). We find it to be very precisely quantized to the value $-1 / 3$. The Berry curvature and the accuracy of the quantization are shown in Fig. 3. We notice that the Berry curvature is a very smooth function of $\varphi_{1}, \varphi_{2}$.

Conclusions. - FCI states are an important recent addition to the arsenal of theoretically predicted, topologically nontrivial states. For these states to be ultimately useful, however, it is imperative to bring them closer to reality. In this Letter, we have presented results that do this in two ways. (i) FCI states are not limited to the energy scale of weak interactions, but can arise for arbitrarily strong repulsion, meaning that the search for such states can be extended to materials with strong correlations. (ii) FCI states are robust against appreciable band mixing and hence candidate systems need not have a very large gap to host them, even though the bands that are mixed have opposite Chern numbers, in contrast to the case of band mixing in Landau levels $[32,33]$. We have based our conclusions on exact numerical evidence obtained with Lanczos diagonalization, that allows us to identify the character and the properties of the ground states on finite systems.

The results of our work reduce the constraints that a material must meet to host a FCI phase of matter. The smoking gun for observing a FCI state in the laboratory is the measurement of a fractional value of the Hall conductivity in a four terminal set up without applied magnetic field. The quantized value of the Hall conductivity is independent of details such as geometry and the microscopic termination of the sample, which would determine the detailed structure of the edge channels. Nevertheless, there remain many material-science challenges. Changing the quantization value of the Hall conductivity depends on the ability to tune the filling fraction. For the conventional quantum Hall effect in semiconductors, one has, in addition to the ability to back gate the sample, the possibility of varying the magnetic field as a way of changing filling fractions. For the known two-dimensional topological band insulators, it is much harder to change doping so as to approach a given commensurate filling fraction. Chemical doping could be used, but it may also introduce strong disorder. These are examples of challenges that remain, but band mixing due to strong interactions, we have established in this Letter, is not one of the main obstacles to realizing a FCI phase of matter.

S. K. was supported by the Deutsche Forschungsgemeinschaft under the Emmy-Noether Program (Grant No. DA 1235/1-1). T. N. acknowledges financial support by the Swiss National Science Foundation. C. C. was supported by DOE Grant No. DEFG02-06ER46316.

[1] E. Tang, J.-W. Mei, and X.-G. Wen, Phys. Rev. Lett. 106, 236802 (2011).

[2] K. Sun, Z. Gu, H. Katsura, and S. Das Sarma, Phys. Rev. Lett. 106, 236803 (2011).

[3] T. Neupert, L. Santos, C. Chamon, and C. Mudry, Phys. Rev. Lett. 106, 236804 (2011).

[4] D. C. Tsui, H. L. Stormer, and A. C. Gossard, Phys. Rev. Lett. 48, 1559 (1982).

[5] N. Y. Yao, A. V. Gorshkov, C. R. Laumann, A. M. Läuchli, J. Ye, and M. D. Lukin, Phys. Rev. Lett. 110, 185302 (2013).

[6] P. Ghaemi, J. Cayssol, D. N. Sheng, and A. Vishwanath, Phys. Rev. Lett. 108, 266801 (2012).

[7] A. G. Grushin, A. Gómez-León, and T. Neupert, arXiv:1309.3571.

[8] D. Xiao, W. Zhu, Y. Ran, N. Nagaosa, and S. Okamoto, Nat. Commun. 2, 596 (2011).

[9] J. W. F. Venderbos, S. Kourtis, J. van den Brink, and M. Daghofer, Phys. Rev. Lett. 108, 126405 (2012).

[10] S. Kourtis, J. W. F. Venderbos, and M. Daghofer, Phys. Rev. B 86, 235118 (2012).

[11] S. A. Parameswaran, R. Roy, and S. L. Sondhi arXiv:1302.6606.

[12] E. J. Bergholtz and Z. Liu, Int. J. Mod. Phys. B 27, 1330017 (2013).

[13] A. G. Grushin, T. Neupert, C. Chamon, and C. Mudry, Phys. Rev. B 86, 205125 (2012).

[14] C. Chamon and C. Mudry, Phys. Rev. B 86, 195125 (2012).

[15] G. Murthy and R. Shankar, Phys. Rev. B 86, 195146 (2012).

[16] A. M. Läuchli, Z. Liu, E. J. Bergholtz, and R. Moessner, Phys. Rev. Lett. 111, 126802 (2013). 
[17] T. Neupert, L. Santos, S. Ryu, C. Chamon, and C. Mudry, Phys. Rev. B 84, 165107 (2011).

[18] B. A. Bernevig and S.-C. Zhang, Phys. Rev. Lett. 96, 106802 (2006).

[19] M. Levin and A. Stern, Phys. Rev. Lett. 103, 196803 (2009).

[20] F. Wang and Y. Ran, Phys. Rev. B 84, 241103(R) (2011).

[21] M. Trescher and E. J. Bergholtz, Phys. Rev. B 86, 241111(R) (2012).

[22] S. Yang, Z.-C. Gu, K. Sun, and S. Das Sarma, Phys. Rev. B 86, 241112(R) (2012).

[23] Y.-F. Wang, H. Yao, C.-D. Gong, and D. N. Sheng, Phys. Rev. B 86, 201101(R) (2012).

[24] Z. Liu, E. J. Bergholtz, H. Fan, and A. M. Läuchli, Phys. Rev. Lett. 109, 186805 (2012).

[25] A. Sterdyniak, C. Repellin, B. A. Bernevig, and N. Regnault, Phys. Rev. B 87, 205137 (2013).
[26] S. Kourtis and M. Daghofer, arXiv:1305.6948.

[27] P. Fendley, K. Schoutens, and J. de Boer, Phys. Rev. Lett. 90, 120402 (2003).

[28] P. Fendley and K. Schoutens, Phys. Rev. Lett. 95, 046403 (2005).

[29] N. G. Zhang and C. L. Henley, Phys. Rev. B 68, 014506 (2003).

[30] N. G. Zhang and C. L. Henley, Eur. Phys. J. B 38, 409 (2004).

[31] See Supplemental Material at http://link.aps.org/ supplemental/10.1103/PhysRevLett.112.126806 for additional information on the characterization of FCI states and the evaluation of $n_{+}$.

[32] A. Sterdyniak, N. Regnault, and G. Möller, Phys. Rev. B 86, 165314 (2012).

[33] M. Hafezi, A. S. Sø rensen, E. Demler, and M. D. Lukin, Phys. Rev. A 76, 023613 (2007). 